\title{
Diagnostic Challenges in Outpatient Stroke: Stroke Chameleons and Atypical Stroke Syndromes
}

\section{Emma JC Wallace (10 \\ Ava L Liberman (iD}

Montefiore Medical Center, Albert Einstein College of Medicine, Department of Neurology, Bronx, NY, USA
Correspondence: Ava L Liberman Montefiore Medical Center, Albert Einstein College of Medicine, Department of Neurology, 3316 Rochambeau Avenue, 4th Floor, Bronx, NY, 10467, USA

Email avliberm@montefiore.org

\begin{abstract}
Failure to diagnose transient ischemic attack (TIA) or stroke in a timely fashion is associated with significant patient morbidity and mortality. In the outpatient or clinic setting, we suspect that patients with minor, transient, and atypical manifestations of cerebrovascular disease are most prone to missed or delayed diagnosis. We therefore detail common stroke chameleon symptoms as well as atypical stroke presentations, broadly review new developments in the study of diagnostic error in the outpatient setting, suggest practical clinical strategies for diagnostic error reduction, and emphasize the need for rapid consultation of stroke specialists when appropriate. We also address the role of psychiatric disease and vascular risk factors in the diagnostic evaluation and treatment of suspected stroke/TIA patients. We advocate incorporating diagnostic timeouts into clinical practice to assure that the diagnosis of TIA or stroke is considered in all relevant patient encounters after a detailed history and examination are conducted in the outpatient setting.
\end{abstract}

Keywords: diagnostic error, stroke chameleons, cerebrovascular disease, stroke

\section{Introduction}

Stroke is the second leading cause of mortality and a major cause of morbidity worldwide. ${ }^{1}$ Failure to correctly diagnose stroke and TIA may preclude initiation of secondary stroke prevention, resulting in adverse patient outcomes. ${ }^{2}$ The efficacy of acute ischemic stroke thrombolysis as well as endovascular thrombectomy are highly time-sensitive. ${ }^{3,4}$ While a great deal of research in stroke misdiagnosis has focused on the emergency setting, ${ }^{5}$ this review explores stroke misdiagnosis in the outpatient setting. Accurate diagnosis of stroke/TIA to prevent stroke chameleons, false-negative stroke cases, is essential to assure appropriate stroke treatment and prevention strategies are initiated regardless of the care setting. While stroke overdiagnosis, stroke mimics or false-positives are other important components of stroke diagnostic error, we will here focus exclusively on the identification and reduction of stroke chameleons. ${ }^{6}$ We will review a number of recent developments in the study of diagnostic error before focusing on the clinical characteristics of stroke chameleons. This narrative review will also detail some atypical cerebrovascular presentations that may be misinterpreted as psychiatric disease and describe relationships between psychiatric illness and stroke/TIA misdiagnosis based on recent data. 


\section{Diagnostic Error}

\section{Definition of Diagnostic Error}

Diagnostic errors have serious consequences for patients and their families. In 2015, the National Academy of Medicine (NAM) of the United States warned that most people will experience a diagnostic error in their lifetime. To advance the field of diagnostic quality and safety, the NAM developed a useful definition of diagnostic error. Diagnostic error is now defined as a failure to: (a) establish an accurate and timely explanation of the patient's health problem(s), or (b) communicate that explanation to the patient. $^{7}$ This new definition includes a number of earlier conceptions of diagnostic error, including wrong, missed, and delayed diagnoses, while encouraging a patient-centric approach. ${ }^{8}$ In acute cerebrovascular disease, delayed diagnoses can be particularly harmful given the time-sensitive nature of many acute treatments. ${ }^{4-9-11}$ Prioritizing research attention towards diseases where diagnostic errors are known to be a substantial cause of patient harms, such as stroke, has substantial potential to improve public health. ${ }^{12}$

\section{Outpatient Diagnostic Error}

The outpatient or clinic setting is a well-known source of medical errors, with an estimated $5.1 \%$ of primary care visits resulting in a misdiagnosis. ${ }^{13}$ In a large study of medical malpractice claims from 1986 to 2010, of the nearly 100,000 US diagnostic error-related claims reviewed, significantly more occurred in the outpatient than inpatient setting $(68.8 \%$ vs $31.2 \%) .{ }^{14}$ In a different study of medical malpractice claims that only included ischemic stroke patients, of the 235 claims that alleged a diagnostic error, only $28 \%$ originated in the outpatient setting. ${ }^{15}$ The frequency with which stroke patients present initially to an outpatient clinic is not known, but among patients with transient ischemic attacks (TIA) or minor strokes we do know that testing and treatment pathways are underdeveloped for those initially evaluated in the outpatient setting. ${ }^{16,17}$

A few studies have used qualitative data to identify sources of diagnostic error in the outpatient or clinic setting. In 2012, a multi-site survey of general internists and family physicians regarding cognitive factors related to missed and delayed diagnosis found that the most common problems reported were "knowledge base inadequate or defective" and "faulty detection or perception" of symptoms or signs. ${ }^{18}$ In a separate study of outpatient providers using focus groups, concerns about health system structure and providers' interactions with one another and with patients far exceeded any discussion of cognitive factors that might affect the diagnostic process. ${ }^{19}$ Though neither study was specific to stroke misdiagnosis, these results suggest that improving outpatient diagnostic processes will require focusing attention on the organization of the health care system as well as on providers' clinical knowledge. Among ischemic stroke patients, a study of malpractice claims found that the most frequent process breakdown was in the initial patient-provider encounter. ${ }^{15}$ To reduce rates of stroke chameleons in the clinic setting, we therefore suspect that improving providers' knowledge of stroke symptoms as well as enhancing their ability to recognize patients' presenting signs or symptoms as due to cerebrovascular disease will aid in the reduction of stroke misdiagnosis-related morbidity and mortality.

\section{Stroke Chameleons}

Ischemic stroke is defined as an episode of neurological dysfunction caused by focal cerebral, spinal, or retinal infarction. To determine whether or not infarction has occurred, either (1) pathological, imaging, or other objective evidence of cerebral, spinal cord, or retinal focal ischemic injury in a defined vascular distribution or (2) clinical evidence of infarction based on symptoms persisting $\geq 24$ hours or until death with other etiologies excluded is needed..$^{20}$ Neuroimaging is an essential component of the diagnostic work-up for any patient with suspected infarction and is necessary to differentiate between ischemic and hemorrhagic stroke. ${ }^{20}$ A number of screening tools to help with prehospital stroke recognition by the community and/or emergency medical services have been developed, but no tool is clearly superior to another. ${ }^{21}$

Most of the well-established prehospital stroke screening tools as well as national stroke education programs focus on identifying typical stroke symptoms such as unilateral weakness, facial droop, or speech difficulties. ${ }^{21-24}$ Stroke chameleons, however, present with atypical stroke symptoms as opposed to hemiparesis, facial droop, or dysarthria. ${ }^{25-27}$ In a recent meta-analysis, missed or delayed (false-negative) diagnosis in the ED occurred in $8.7 \%$ of ischemic stroke, TIA, and subarachnoid hemorrhage patients. ${ }^{5}$ Common presenting symptoms among hospitalized stroke chameleons include dizziness/vertigo, nausea/vomiting, confusion, decreased level of consciousness, altered mental status, generalized weakness, and severe hypertension. ${ }^{26-29}$ Among hospitalized stroke patients, chameleons are both more likely to be those 
with minor symptoms and those with very severe symptoms as compared to correctly diagnosed stroke patients. ${ }^{2}$ Stroke misdiagnosis in the acute setting is more likely to occur among younger stroke patients. ${ }^{2,27}$

It is unlikely that patients with symptoms that require urgent evaluation, such as severe sensorimotor deficits, decreased level of consciousness, or profound confusion, will be initially evaluated in an outpatient or clinic setting. However, patients with subacute minor or transient neurological symptoms, including dizziness, may present to clinic. Ischemic stroke is preceded by a TIA in $20-25 \%$ of patients ${ }^{30}$ and, among patients with posterior circulation infarctions, rates of transient neurological events in the days preceding infarction are particularly high. ${ }^{31}$ Important predictors of stroke/TIA include definite focal symptoms, that the patient was well in the last week, nongradual symptom onset, and the ability to determine a precise time of symptom onset. ${ }^{32,33}$

When assessing patients with confusion, altered mental status, or speech abnormalities in clinic, obtaining collateral history as to whether or not symptom onset was sudden is of critical importance (Table 1). Right middle cerebral artery strokes can manifest as an acute confusional state or an acute onset delirium often without other obvious signs of cerebral infarction. ${ }^{34}$ Similarly, thalamic infarcts can result in a variety of behavioral abnormalities. ${ }^{35}$

While sudden symptom onset is associated with true stroke diagnosis, ${ }^{32,33}$ once cerebral ischemia occurs patients can experience worsening in their clinically defined symptoms due to stroke-in-progression, a phenomena which occurs during the hours following the index event. ${ }^{36}$ An essential component of stroke/TIA diagnostic evaluation is assessing cervical-cranial vasculature, as large vessel disease is associated with increased risk of early stroke recurrence and clinical worsening (Figure 1). ${ }^{37-39}$ In particular, basilar artery occlusion (BAO) portends high rates of morbidity and mortality. ${ }^{40}$ Patients with BAO can present with altered mental status or TIA and progress to coma. ${ }^{31,41}$ Syncope, a sudden loss of consciousness followed by spontaneous recovery can be confused with BAO. While alteration in consciousness can occur in both syncope and BAO, alterations due to syncope rapidly resolve whereas BAO causes ischemia to the reticular activating system resulting in progressive decreases in alertness. ${ }^{42,43}$

\section{Dizziness}

Dizziness is one of the most common presenting symptoms among stroke chameleons evaluated emergently. ${ }^{5}$

Table I Summary of Possible Stroke Chameleons and Some Atypical Stroke Symptom Presentations

\begin{tabular}{|c|c|c|}
\hline $\begin{array}{l}\text { Patient } \\
\text { Presentation }\end{array}$ & Key Elements of History & $\begin{array}{l}\text { Key Elements of Physical } \\
\text { Examination }\end{array}$ \\
\hline Altered mental status & Symptom onset, duration, fluctuation, presence of abnormal movements & $\begin{array}{l}\text { Mental status exam (including level of } \\
\text { alertness), motor exam }\end{array}$ \\
\hline Dizziness & $\begin{array}{l}\text { Triage (abnormal vital signs, other prominent associated features), timing } \\
\text { (episodic, acute, chronic), triggers and exposures }\end{array}$ & Targeted eye movements \\
\hline Headache & $\begin{array}{l}\text { History of prior headache, associate features (weakness, vision changes), } \\
\text { abrupt or insidious onset, aura, trauma history }\end{array}$ & $\begin{array}{l}\text { Neurological examination including } \\
\text { fundoscopy }\end{array}$ \\
\hline Wrist drop & Timing of wrist drop, history of trauma & $\begin{array}{l}\text { Motor and sensory exam, fist closure } \\
\text { maneuver }\end{array}$ \\
\hline Foot drop & Timing of foot drop, history of trauma & Motor and sensory exam, foot tapping \\
\hline $\begin{array}{l}\text { Hyperkinetic } \\
\text { movement disorder }\end{array}$ & Onset of abnormal movement, associated symptoms & Motor exam including tone \\
\hline Alien limb syndrome & $\begin{array}{l}\text { Onset of phenomenon, ability to control movement, associated cognitive } \\
\text { changes }\end{array}$ & Mental status exam, motor exam \\
\hline $\begin{array}{l}\text { Visual hallucinations or } \\
\text { confabulations }\end{array}$ & $\begin{array}{l}\text { Psychiatric history, associated neurological deficits, timing of symptom onset, } \\
\text { insight }\end{array}$ & $\begin{array}{l}\text { Language exam (including naming), } \\
\text { visual field testing }\end{array}$ \\
\hline
\end{tabular}




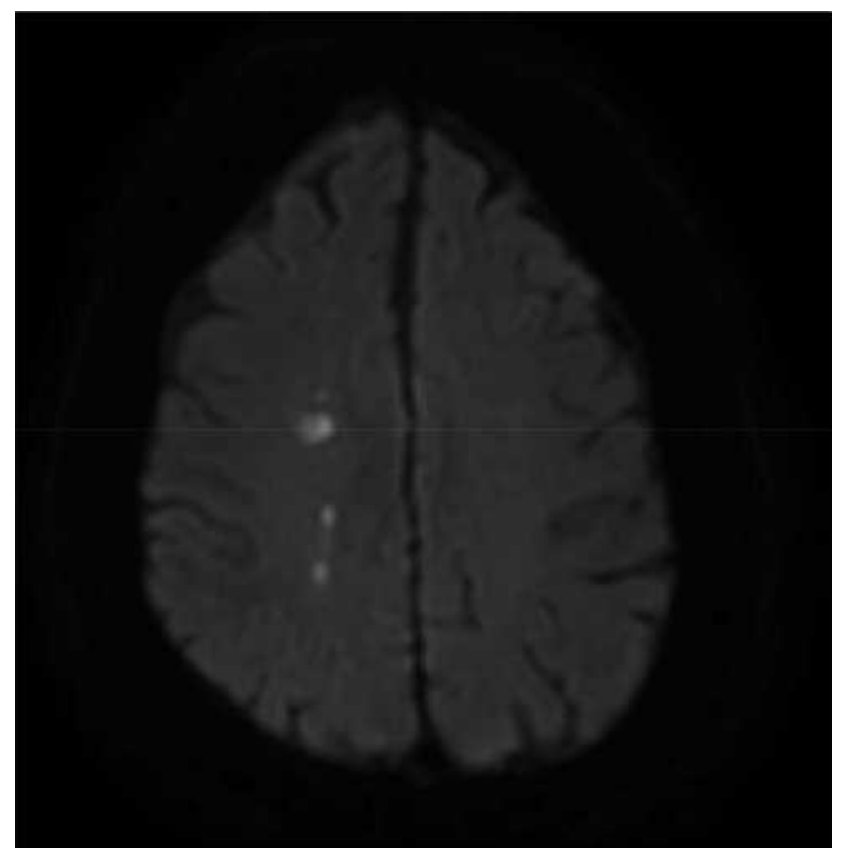

Figure I Illustrative stuttering symptom case.

Notes: An 8I-year-old man with a history of hypertension, diabetes, and hyperlipidemia who presented initially with left lower extremity weakness who was then noted to have transient left arm weakness and left-sided facial droop once admitted. Vessel imaging revealed critical right internal carotid artery stenosis. His MR brain diffusion weighted imaging sequence demonstrated acute watershed infarcts in the right centrum semiovale.

However, in the outpatient setting, dangerous neurological conditions are an infrequent cause of dizziness complaints. ${ }^{4,45}$ Physicians traditionally seek to differentiate inner ear conditions from stroke or other central causes of vertigo when evaluating dizziness by focusing on symptom quality or the "type" of dizziness (eg vestibular versus neurological versus cardiac). This traditional approach to dizziness evaluation is flawed and may be a source of diagnostic error. ${ }^{46,47}$ A newer approach to evaluate emergency dizziness complaints has been suggested by experts in vestibular disease and diagnostic error dubbed TiTRATE. ${ }^{48}$ This approach focuses on the onset, duration, and evolution (timing) of dizziness as well as triggers (actions, movements, or situations) that provoke dizziness. It is important to note that, to correctly use TiTRATE, clinicians must clearly distinguish between dizziness triggers (head or body motion that provokes new symptoms not previously present) from exacerbating features (head or body motion that worsens pre-existing dizziness) since the latter can occur in many different types of dizziness. ${ }^{48}$

Focusing on dizziness timing and triggers can be used to categorize dizzy patients into one of four categories according to the TiTRATE approach: (1) triggered episodic, (2) spontaneous episodic, (3) post-exposure acute, and (4) spontaneous acute. Episodic vestibular syndrome involves transient dizziness lasting seconds, minutes, or hours. When the episodic vestibular syndrome is triggered, prototypical causes include benign paroxysmal peripheral vertigo (BPPV) or orthostatic hypotension whereas when the episodic vestibular syndrome is spontaneous the cause is often vestibular migraine, vasovagal syncope, or panic attacks. Some TIA patients can present with complaints of the spontaneous episodic vestibular syndrome. $^{46,48}$

When dizziness is due to an acute ischemic stroke, the typical presentation is as an acute vestibular syndrome without a clear preceding exposure or symptom trigger. The acute vestibular syndrome is defined as acute-onset, continuous dizziness, vertigo or unsteadiness lasting days to weeks and generally includes features suggestive of new, ongoing vestibular system dysfunction such as vomiting, nystagmus, or postural instability (Figure 2). Vestibular neuritis is a common benign cause of the acute vestibular syndrome. Particular exam maneuvers, including the Head Impulse-Nystagmus-Test of Skew (HINTS) test where the presence of any of three oculomotor signs (normal horizontal head impulse, gaze-direction nystagmus, or skew deviation) suggests a central cause, and advanced imaging studies are indicated to aide with diagnosis once the category of dizziness is determined. ${ }^{48,49}$ Extending the TiTRATE approach to the clinic setting may prove useful in identifying TIA or stroke patients among those who present with dizziness.

\section{Minor Stroke}

Minor stroke patients, stroke with minimal or subtle deficits, are frequently misdiagnosed when they present emergently. $^{2,5}$ Failure to accurately diagnose the symptoms or signs of minor stroke in the ED may be partially due to that setting's fast pace and emphasis on critical illness. ${ }^{50,51}$ There may be more time to examine patients with neurological complaints in the clinic setting so that subtle or minor neurological deficits suggestive of cerebral infarction can be detected. Taking time to conduct a detailed neurological examination may improve stroke diagnostic accuracy. Suspected minor stroke patients seen in clinic should be quickly referred for urgent stroke evaluations as they are at risk of stroke recurrence. ${ }^{52}$ Like all stroke patients, minor stroke patients require a diagnostic evaluation to identify their stroke mechanism to 

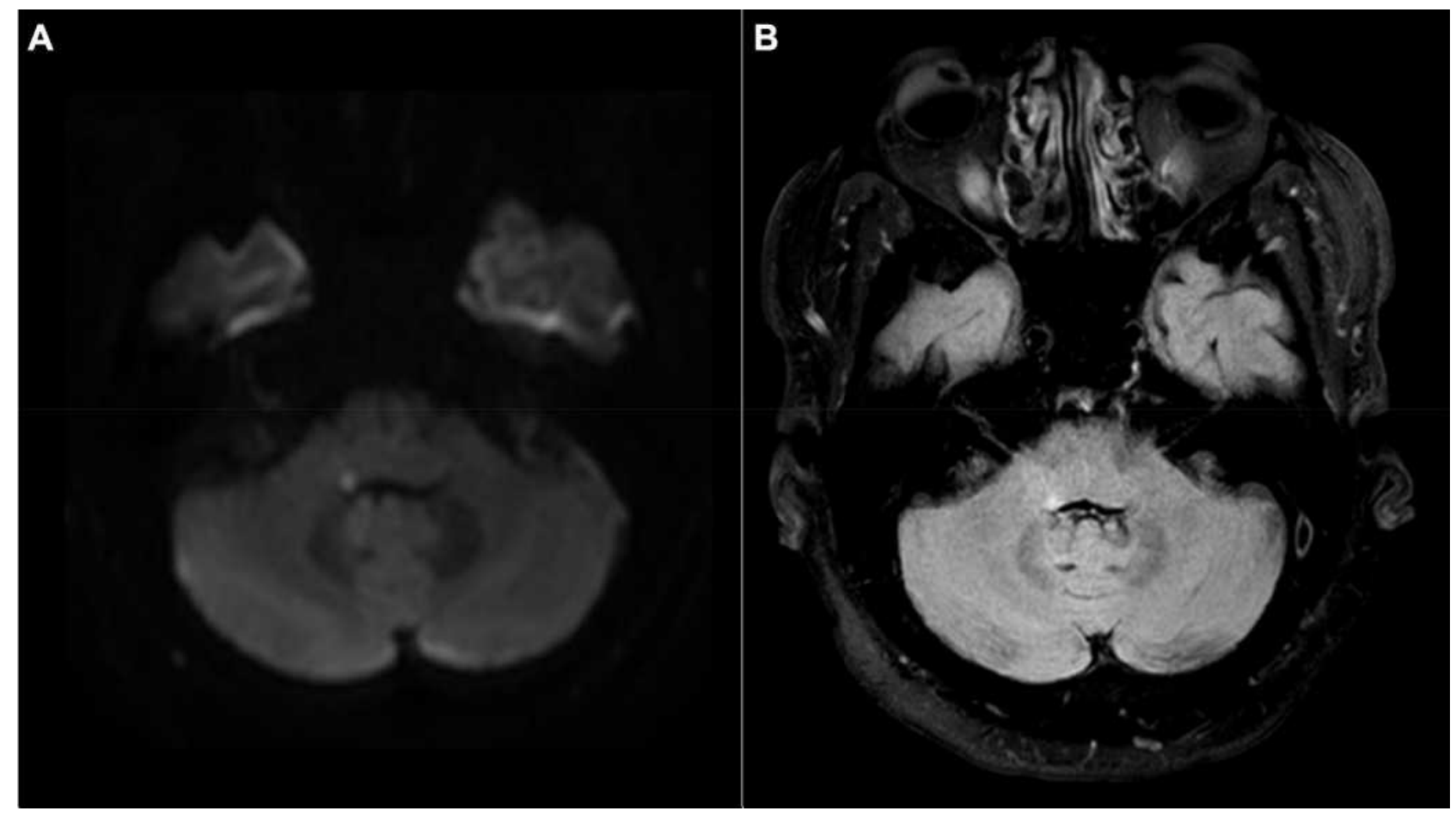

Figure 2 Illustrative stroke chameleon case.

Notes: A 59-year-old man with a history of hypertension who presented with dizziness and feeling "off-balance" for a few days. His index evaluation did not include an evaluation of eye movements, coordination, or gait. The patient's vital signs were notable for elevated systolic blood pressure and, therefore, he was admitted to the hospital. On hospital day two, the patient continued to complain of disequilibrium. Neurological examination found right beating horizontal nystagmus and an ataxic gait. MRI brain found a subacute right middle cerebellar peduncle infarct seen on diffusion weighted imaging sequence (panel $\mathbf{A}$ ), which corresponded to hyperintensity seen on fluidattenuated inversion recovery (FLAIR) sequence (panel B). The apparent diffusion coefficient (ADC) sequence did not clearly show restricted diffusion.

ensure appropriate secondary stroke prevention is initiated. $^{53}$

Occasionally, small infarcts can be misinterpreted as peripheral neuropathies. ${ }^{2}$ In particular, infarction of the precentral gyrus in the region of the cortical hand knob can produce a wrist drop that can be misdiagnosed as a radial nerve palsy. Central versus peripheral causes of wrist drop can be differentiated by asking the patient to perform fist closure of the hand in question. Patients with a true radial nerve palsy will have worsening of their wrist drop, whereas those with a central lesion will demonstrate an elevation of the hand due to synkinetic contraction of the extensor muscles of the forearm. ${ }^{54}$ Foot drop, most commonly caused by peroneal nerve injury around the fibular head, can be caused by a stroke in the high frontal precentral gyrus. Acute onset of foot drop rather than one with insidious onset should clue the examiner in to a potential central cause. ${ }^{55,56}$ On examination, patients with stroke that have affected the corticospinal tract may have a positive Babinski sign; however, the inter-rater reliability of this sign is only fair. ${ }^{57}$ Slowed foot tapping is an upper motor neuron sign that can be more reliably assessed. ${ }^{57,58}$

\section{Transient Ischemic Attack}

TIA patients are at substantial risk of subsequent stroke, with the highest risk in the first few days after their index event. ${ }^{52}$ Modern rates of stroke after TIA range from $1.5 \%$ at 2 days post-TIA to $9.5 \%$ at 5 years after the index event. 52,59 A TIA is defined as a "transient episode of neurological dysfunction caused by focal brain, spinal cord, or retinal ischemia, without acute infarction." ${ }^{60}$ The definition of TIA is no longer timebased, ${ }^{60}$ though transient ischemic attacks usually last for seconds or minutes and are rarely longer than 1 hour. ${ }^{61}$ Determining when a transient neurological complaint is due to cerebrovascular disease can be challenging. ${ }^{62}$ In a recent prospective study of 1028 patients with low-risk transient and minor neurological symptoms, the final diagnosis was revised in $30 \%$ of patients after brain magnetic resonance (MR) imaging was obtained. ${ }^{63}$ Obtaining advanced neuroimaging with MR diffusion weighted imaging (DWI) sequence is increasingly recognized as an essential component in the diagnostic evaluation of TIA and minor stroke patients. $^{60}$ 
Clinical symptoms most suggestive of a definite TIA include: motor weakness in two limbs or in one limb and the face, sensory deficit in two limbs or in one limb and the face, homonymous hemianopia, monocular blindness (amaurosis fugax), and aphasia or dysarthria. Other neurological symptoms, including vertigo and gait instability, can also be due to TIA. ${ }^{64,65}$ As noted above, complaints of vertigo among TIA patients are often that of the spontaneous episodic vestibular syndrome. ${ }^{46,48}$ If a TIA is diagnosed or strongly suspected in the outpatient setting, urgent referral to a TIA clinic or evaluation by a specialist in an ED should be done.

Obtaining a detailed history is key to TIA diagnosis. Close attention should be paid to elements predictive of non-vascular causes of transient events, including gradual symptom onset, prior history of unexplained transient neurological attack, and presence of additional nonspecific symptoms. ${ }^{33}$ Distinguishing migraine headaches from TIA when focal symptoms are reported can be challenging and, given increased risk of stroke among patients with headache complaints, erring on the side of rapid expert evaluation in cases of diagnostic uncertainty is advisable. ${ }^{66}$ Recently, criteria have been developed to help differentiate migraine with aura from TIA in the acute setting, called the explicit diagnostic criteria for TIA (EDCT) ${ }^{67}$ The EDCT contains 5 criteria for the diagnosis of TIA that touch on the fact that sudden symptom onset, lack of irritative symptoms (eg photopsias, pins and needles), and no headache accompanying or following the neurological symptoms within 1 hour are more typical of TIA than migraine with aura. ${ }^{67,68}$

\section{Headache}

The relationship between stroke and headache, particularly migraine headache, is complex. ${ }^{66}$ Though the vast majority of headache visits in the emergency setting are not due to dangerous conditions, ${ }^{69}$ among patients with TIA and minor strokes headache at symptom onset is estimated to occur in nearly $18 \%$ of patients. ${ }^{70}$ These patients with subtle or transient neurological symptoms who complain of a headache may be particularly prone to misdiagnosis. Using administrative data from multiple states, a preceding ED visit for headache resulting in discharge to home (ED treat-and-release visit) has been noted in a non-trivial amount of patients hospitalized for stroke. ${ }^{71}$ Similarly, a study using administrative claims data from six states estimated that around $0.5 \%$ of all ED headache patients may be misdiagnosed at index ED visit, as evidenced by a hospitalization for dangerous neurological disease shortly after their index ED visit. ${ }^{72}$ Rates of stroke misdiagnosis among patients who complain of headache in the outpatient setting are, to our knowledge, unknown.

As a first step, differentiating between cases where headache and stroke are in close temporal proximity versus at separate times is useful. ${ }^{73,74}$ Headache at the same time or in close temporal proximity to acute ischemic stroke or TIA symptoms is most likely to result in an erroneous false-negative stroke diagnosis. Headaches can be precipitated by stroke/TIA, stroke/TIA can trigger a migraine attack, a migraine attack may trigger a stroke/ TIA, and, rarely, strokes can occur during the course of a typical migraine with aura attack (eg migrainous infarction) ${ }^{73-75}$ It is also important to keep in mind that, albeit rarely, a single underlying process, such as small vessel arteriopathies (eg cerebral autosomal dominant arteriopathy with subcortical infarcts and leukoencephalopathy [CADASIL] $)^{76}$ or vasculitis involving the central nervous system, ${ }^{77}$ can cause headaches and cerebrovascular disease, as well as the fact that migraine, particularly with aura, is a risk factor for stroke. ${ }^{78}$ A key component of identifying cerebral ischemia among patients with headache is the presence of focal neurological deficits on exam or report of transient focal deficits consistent with a TIA. Additionally, patients with a recent ED treat-and-release visit for "benign" headache who had a normal head CT and those with a history of prior stroke or TIA who complain of headache may be at increased risk of shortterm stroke; ${ }^{79,80}$ a higher level of suspicion for cerebrovascular ischemia in these patient subgroups is likely warranted.

\section{Atypical Stroke Syndromes}

While the aforementioned conditions are well-known presentations of stroke chameleons that may be seen in the outpatient setting, there are other uncommon manifestations of cerebrovascular disease that can present significant diagnostic challenges to the non-specialist. We therefore detail some of these atypical stroke syndromes here.

\section{Hyperkinetic Movement Disorders}

Most stroke and TIA patients, including stroke chameleons, present with loss of function (weakness, numbness) or "negative" symptoms. Rarely, patients with cerebrovascular ischemia can present with "positive" symptoms (shaking, paresthesia). Hyperkinetic movement disorders (HMD) are defined as abnormal, repetitive, involuntary 
movements that are classified as dystonia, chorea/ballism, or tremor. They are estimated to occur in $1 \%$ of all acute stroke presentations. ${ }^{56}$ Given the rarity of stroke causing HMD, stroke patients with these symptoms are likely prone to misdiagnosis. To our knowledge, however, specific rates of misdiagnosis in this subgroup of stroke patients has not been reported. HMD can occur after infarcts in the putamen, subthalamic nucleus, caudate, or the posterior nucleus of the thalamus. Chorea/ballism can present at stroke onset whereas tremor more typically occurs at least 1 month after initial cerebral insult. ${ }^{81}$ For the medical practitioner, the index of suspicion for a cerebrovascular cause of a new HMD should be high if the patient is known to have medical comorbidities that put them at risk of cerebrovascular events including hypertension, diabetes, atrial fibrillation, prior stroke, or hyperlipidemia. ${ }^{81,82}$ A complaint of repetitive involuntary tremor triggered by an orthostatic position change or exercise is suggestive of limb-shaking TIA rather than seizure or an HMD. ${ }^{83}$ Limb-shaking TIAs require emergent evaluation, as they are associated with decreased cerebral perfusion in the setting of high-grade large artery stenosis, most frequently of the carotid artery. ${ }^{83-85}$ Similarly, lightinduced monocular vision loss can reflect decreased cerebral perfusion due to carotid artery disease and also warrants emergent evaluation. ${ }^{86}$

\section{Alien Limb Syndrome}

Alien limb syndrome is described as involuntary, complex, and purposeful movements of a limb with an accompanying sense that the limb is foreign. A case series examining etiologies of alien limb syndrome which included 150 patients found that $10 \%$ of patients had a vascular etiology. ${ }^{87}$ Alien limb syndrome usually involves the upper extremity; however, cases have been reported involving the lower extremity. ${ }^{88}$ Patients with damage to the supplementary motor area, anterior cingulate gyrus, medial prefrontal cortex, and anterior corpus callosum of the dominant hemisphere demonstrate reflexive grasping, groping, and manipulations; this is termed frontal alien hand syndrome. ${ }^{89}$ Alternatively, antagonistic action between the hands, extremity levitation, and uncontrolled grasping generally occur after lesions of the anterior corpus callosum. ${ }^{89}$ Alien limb syndrome is most commonly caused by corticobasal syndrome, a multisystem degenerative disorder manifesting with dystonia, akinesia, and rigidity. ${ }^{90}$ Given its rarity, alien limb syndrome has been misdiagnosed as myelopathy, stiff limb syndrome, or as a functional disorder. ${ }^{88}$

\section{Anton's Syndrome}

Patients with bilateral occipital lobe infarcts can present with Anton's syndrome, which is a constellation of denial of blindness and confabulation of visual sensory information. ${ }^{91}$ These patients fabricate imaginary information to compensate for a loss of occipital lobe processing and/or a disconnection from relevant association areas due to stroke. ${ }^{92}$ Important clues to the diagnosis of Anton's syndrome are quickly ascertained by simple examination and observation: patients with Anton's syndrome lack insight into their disorder, will incorrectly name items presented to them, demonstrate an inability to reproduce drawings, and have impaired spatial navigation. ${ }^{91,93}$

\section{Charles Bonnet Syndrome}

Charles Bonnet Syndrome (CBS) is referred to as "phantom vision" and consists of visual hallucinations which occur after loss of input into the occipital cortex. $^{94,95}$ Strokes resulting in visual field cuts typically in the occipital or temporal lobe, can result in CBS. Patients with this syndrome have described seeing well-formed animals, grid-like patterns, and even shrubbery. ${ }^{94,95}$ A patient's history can provide critical clues in differentiating CBS from a primary psychiatric disorder: patients with CBS have intact cognition and insight into the notion that their hallucinations are not real. $^{96}$ Patients with CBS initially report discomfort associated with their hallucinations; however, with habituation their discomfort declines. ${ }^{95}$

\section{Gerstmann Syndrome}

Gerstmann syndrome is a rare stroke syndrome which consists of a tetrad of symptoms: agraphia, acalculia, finger agnosia, and right-left confusion. ${ }^{97}$ Lesions responsible for Gerstmann syndrome are most commonly localized to the angular gyrus of the dominant hemisphere, with subcortical extension. ${ }^{98}$ There has been some controversy over the entity as patients can have lesions of the angular gyrus with intact writing capability termed "incomplete" Gerstmann syndrome. Upon further examination of lesion localization in these patients, patients with "incomplete" Gerstmann syndrome do not have extension of the lesion into the superior parietal area. ${ }^{98}$ 


\section{Psychiatric Disease}

In addition to some stroke chameleons being incorrectly diagnosed as psychiatric disease, ${ }^{2}$ patients with true psychiatric illness may be at increased risk of stroke misdiagnosis. In a study of ischemic stroke patients, the presence of comorbid psychiatric disease was associated with $20 \%$ lower odds of receiving acute thrombolysis in the ED as compared to patients without psychiatric illness. ${ }^{99}$ The reasons for this treatment disparity are uncertain. Causes may include delayed arrival at the ED, difficult to obtain history, or initial incorrect diagnosis by providers. ${ }^{99}$ Based on current data, assuring relevant collateral information from the treating psychiatrist is quickly relayed to emergency medicine providers and/or consulting vascular neurologists may be particularly helpful to facilitate thrombolysis for eligible stroke patients with psychiatric disease. Another important point regarding stroke among patients with psychiatric illness is that an increased risk of stroke has been noted in the year following psychiatric hospitalizations. ${ }^{100}$ Among patients with a prior ED visit or inpatient stay for psychiatric disease, the highest odds of stroke occurs within 15 days of that initial psychiatric visit. ${ }^{100}$ While the cause of this increased stroke risk is uncertain, it suggests psychosocial stress may serve as a stroke trigger and may point to potential diagnostic error at index visit wherein a cerebrovascular event is misdiagnosed as psychiatric in nature. ${ }^{100,101}$ Having an increased level of suspicion for stroke in clinic patients with neurological symptoms or signs who have had a recent ED visit or inpatient stay due to psychiatric illness may be warranted.

\section{Diagnostic Error Reduction}

Well-established interventions to improve diagnostic accuracy in the outpatient setting are limited. There has long been an interest in identifying and combatting common cognitive biases (anchoring, framing effect) to reduce cognitive errors that lead to misdiagnosis. ${ }^{102}$ Cognitive errors may be particularly germane to cases of stroke misdiagnosis as these patients frequently have signs or symptoms that are atypical for stroke or are non-specific. ${ }^{6,50}$ To date, however, well-done educational programs to train learners on cognitive de-biasing strategies have not been effective.103 Additionally, a study evaluating experienced physicians using clinical case vignettes found very poor interrater reliability regarding the presence or absence of individual cognitive biases. ${ }^{104}$
A simple practical tool to avoid cognitive error may be for providers to take a moment to pause to reflect on the plausibility of the working diagnosis, a diagnostic "timeout." 105 During a diagnostic time-out, providers can ask themselves "Why can't this be something else?", consider what would happen if the working diagnosis is wrong, as well as apply any indicated clinical decision support. A diagnostic time-out can be augmented by checklists of potential alternative diagnoses (differential diagnosis checklists). ${ }^{105-107}$ In the context of improving diagnostic accuracy in stroke, simply pausing to reflect on whether or not the presenting symptoms or signs could be vascular in origin may be impactful. As yet, there is no robust data in support of diagnostic time-outs. A pilot randomized controlled clinical trial found that having physicians perform diagnostic time-outs with a differential diagnosis checklist did not reduce diagnostic error overall, but did reduce error in the subgroup of ED physicians. ${ }^{108}$ Similarly, in a mixed-methods study of primary care providers, taking a diagnostic time-out after clinical encounters led to a different action in $13 \%$ of cases but did not clearly reduce diagnostic errors. Participants reported that taking a diagnostic time-out was brief and well-integrated into their work flow. ${ }^{109}$

Real-time diagnostic time-outs have the potential to meaningfully reduce diagnostic error, though additional evidence is needed. Given that diagnostic time-outs are a low-risk, easily adoptable approach to improving diagnostic accuracy, their incorporation into routine outpatient clinical practice should be strongly considered. Reflecting on whether stroke or TIA could be the cause of any of the presenting complaints known to be frequent among stroke chameleons may reduce stroke misdiagnosis. However, diagnostic time-outs are best suited to when subacute or chronic symptoms are being evaluated since, if the patient has symptoms suspicious for an acute cerebrovascular event, any delay in urgent evaluation can preclude timesensitive treatments.

\section{Conclusion}

Failure to diagnose stroke and TIA in a timely manner is an important source of misdiagnosis-related patient harms. In addition to obtaining a history focused on predictors of stroke/TIA and performing a detailed neurological examination when indicated, a practical tool that providers can immediately incorporate into their practice is to reflect in real-time on the plausibility of the working diagnosis. Among patients with minor and atypical neurological 
complaints, particularly when initial symptom onset is sudden, considering the possibility of cerebrovascular disease as a potential cause may improve stroke/TIA recognition. All suspected stroke and TIA patients should be promptly referred for evaluation and treatment by stroke specialists as many secondary stroke prevention treatments are time-sensitive.

\section{Funding}

NIH research grant K23NS107643 supports Dr. Liberman.

\section{Disclosure}

Neither author reports any relevant conflict of interest disclosures for this work.

\section{References}

1. Katan M, Luft A. Global burden of stroke. Semin Neurol. 2018;38 (2):208-211. doi:10.1055/s-0038-1649503

2. Richoz B, Hugli O, Dami F, Carron PN, Faouzi M, Michel P. Acute stroke chameleons in a university hospital: risk factors, circumstances, and outcomes. Neurology. 2015;85(6):505-511. doi:10.1212/WNL.0000000000001830

3. Lees KR, Bluhmki E, von Kummer R, et al. Time to treatment with intravenous alteplase and outcome in stroke: an updated pooled analysis of ECASS, ATLANTIS, NINDS, and EPITHET trials. Lancet. 2010;375(9727):1695-1703. doi:10.1016/S01406736(10)60491-6

4. Saver JL, Goyal M, van der Lugt A, et al. Time to treatment with endovascular thrombectomy and outcomes from ischemic stroke: a meta-analysis. JAMA. 2016;316(12):1279-1288. doi:10.1001/ jama.2016.13647

5. Tarnutzer AA, Lee S-H, Robinson KA, Wang Z, Edlow JA. ED misdiagnosis of cerebrovascular events in the era of modern neuroimaging. Neurology. 2017;88(15):1468-1477. doi:10.1212/ WNL.0000000000003814

6. Liberman AL, Prabhakaran S. Stroke chameleons and stroke mimics in the emergency department. Curr Neurol Neurosci Rep. 2017;17(2):15. doi:10.1007/s11910-017-0727-0

7. Ball JR, Balogh E. Improving diagnosis in health care: highlights of a report from the national academies of sciences, engineering, and medicine. Ann Intern Med. 2016;164(1):59-61. doi:10.7326/ M15-2256

8. Graber ML. The IOM report on improving diagnosis: new concepts. Diagnosis. 2015;2(4):201-203. doi:10.1515/dx-2015-0029

9. Saver JL, Fonarow GC, Smith EE, et al. Time to treatment with intravenous tissue plasminogen activator and outcome from acute ischemic stroke. JAMA. 2013;309(23):2480-2488. doi:10.1001/ jama.2013.6959

10. Colton K, Richards CT, Pruitt PB, et al. Early stroke recognition and time-based emergency care performance metrics for intracerebral hemorrhage. J Stroke Cerebrovasc Dis. 2020;29(2):104552. doi:10.1016/j.jstrokecerebrovasdis.2019.104552

11. Rothwell PM, Giles MF, Chandratheva A, et al. Effect of urgent treatment of transient ischaemic attack and minor stroke on early recurrent stroke (EXPRESS study): a prospective populationbased sequential comparison. Lancet. 2007;370(9596):14321442. doi:10.1016/S0140-6736(07)61448-2
12. Newman-Toker DE, Schaffer AC, Yu-Moe CW, et al. Serious misdiagnosis-related harms in malpractice claims: the "Big Three" - vascular events, infections, and cancers. Diagnosis (Berl). 2019;6(3):227-240. doi:10.1515/dx-2019-0019

13. Singh H, Meyer AN, Thomas EJ. The frequency of diagnostic errors in outpatient care: estimations from three large observational studies involving US adult populations. BMJ Qual Saf. 2014;23(9):727-731. doi:10.1136/bmjqs-2013-002627

14. Saber Tehrani AS, Lee H, Mathews SC, et al. 25-Year summary of US malpractice claims for diagnostic errors 1986-2010: an analysis from the National Practitioner Data Bank. BMJ Qual Saf. 2013;22(8):672-680. doi:10.1136/ bmjqs-2012-001550

15. Liberman AL, Skillings J, Greenberg P, Newman-Toker DE, Siegal D. Breakdowns in the initial patient-provider encounter are a frequent source of diagnostic error among ischemic stroke cases included in a large medical malpractice claims database. Diagnosis. 2020;7(1):37-43. doi:10.1515/dx-20190031

16. Goldstein LB, Bian J, Samsa GP, Bonito AJ, Lux LJ, Matchar DB. New transient ischemic attack and stroke: outpatient management by primary care physicians. Arch Intern Med. 2000;160 (19):2941-2946. doi:10.1001/archinte.160.19.2941

17. Davey AR, Lasserson DS, Levi CR, et al. Management of transient ischemic attacks diagnosed by early-career general practitioners: a cross-sectional study. Int J Stroke. 2018;13(3):313-320. doi: $10.1177 / 1747493017743053$

18. Sarkar U, Bonacum D, Strull W, et al. Challenges of making a diagnosis in the outpatient setting: a multi-site survey of primary care physicians. BMJ Qual Saf. 2012;21(8):641-648. doi:10.1136/bmjqs-2011-000541

19. Sarkar U, Simchowitz B, Bonacum D, et al. A qualitative analysis of physician perspectives on missed and delayed outpatient diagnosis: the focus on system-related factors. Jt Comm J Qual Patient Saf. 2014;40(10):461. doi:10.1016/s1553-7250(14) 40059-x

20. Sacco RL, Kasner SE, Broderick JP, et al. An updated definition of stroke for the 21st century: a statement for healthcare professionals from the American Heart Association/American Stroke Association. Stroke. 2013;44(7):2064-2089. doi:10.1161/ STR.0b013e318296aeca

21. Rudd M, Buck D, Ford GA, Price CI. A systematic review of stroke recognition instruments in hospital and prehospital settings. Emerg Med J. 2016;33(11):818-822. doi:10.1136/ emermed-2015-205197

22. Aroor S, Singh R, Goldstein LB. BE-FAST (balance, eyes, face, arm, speech, time): reducing the proportion of strokes missed using the FAST mnemonic. Stroke. 2017;48(2):479-481. doi:10.1161/STROKEAHA.116.015169

23. Nordanstig A, Asplund K, Norrving B, Wahlgren N, Wester P, Rosengren L. Impact of the Swedish National Stroke Campaign on stroke awareness. Acta Neurol Scand. 2017;136(4):345-351. doi:10.1111/ane.12777

24. Hickey A, Mellon L, Williams D, Shelley E, Conroy RM. Does stroke health promotion increase awareness of appropriate behavioural response? Impact of the face, arm, speech and time (FAST) campaign on population knowledge of stroke risk factors, warning signs and emergency response. Eur Stroke J. 2018;3 (2):117-125. doi:10.1177/2396987317753453

25. Venkat A, Cappelen-Smith C, Askar S, et al. Factors associated with stroke misdiagnosis in the emergency department: a retrospective case-control study. Neuroepidemiology. 2018;51(34):123-127. doi:10.1159/000491635 
26. Lever NM, Nystrom KV, Schindler JL, Halliday J, Wira C, Funk M. Missed opportunities for recognition of ischemic stroke in the emergency department. J Emerg Nurs. 2013;39(5):434-439. doi:10.1016/j.jen.2012.02.011

27. Arch AE, Weisman DC, Coca S, Nystrom KV, Wira CR, Schindler JL. Missed ischemic stroke diagnosis in the emergency department by emergency medicine and neurology services. Stroke. 2016;47 (3):668-673. doi:10.1161/STROKEAHA.115.010613

28. Madsen TE, Khoury J, Cadena R, et al. Potentially missed diagnosis of ischemic stroke in the emergency department in the Greater Cincinnati/Northern Kentucky Stroke Study. Acad Emerg Med. 2016;23(10):1128-1135. doi:10.1111/acem.13029

29. Dupre CM, Libman R, Dupre SI, Katz JM, Rybinnik I, Kwiatkowski T. Stroke chameleons. J Stroke Cerebrovasc Dis. 2014;23(2):374378. doi:10.1016/j.jstrokecerebrovasdis.2013.07.015

30. Rothwell PM, Warlow CP. Timing of TIAs preceding stroke: time window for prevention is very short. Neurology. 2005;64(5):817820. doi:10.1212/01.WNL.0000152985.32732.EE

31. Paul NL, Simoni M, Rothwell PM. Transient isolated brainstem symptoms preceding posterior circulation stroke: a populationbased study. Lancet Neurol. 2013;12(1):65-71. doi:10.1016/ S1474-4422(12)70299-5

32. Hand PJ, Kwan J, Lindley RI, Dennis MS, Wardlaw JM. Distinguishing between stroke and mimic at the bedside: the brain attack study. Stroke. 2006;37(3):769-775. doi:10.1161/01. STR.0000204041.13466.4c

33. Prabhakaran S, Silver AJ, Warrior L, McClenathan B, Lee VH. Misdiagnosis of transient ischemic attacks in the emergency room. Cerebrovasc Dis. 2008;26(6):630-635. doi:10.1159/ 000166839

34. Mori E, Yamadori A. Acute confusional state and acute agitated delirium. Occurrence after infarction in the right middle cerebral artery territory. Arch Neurol. 1987;44(11):1139-1143. doi:10.1001/archneur.1987.00520230029009

35. Schmahmann JD. Vascular syndromes of the thalamus. Stroke. 2003;34(9):2264-2278. doi:10.1161/01. STR.0000087786.38997.9E

36. Gautier JC. Stroke-in-progression. Stroke. 1985;16(4):729-733. doi:10.1161/01.STR.16.4.729

37. Yaghi S, Rostanski SK, Boehme AK, et al. Imaging parameters and recurrent cerebrovascular events in patients with minor stroke or transient ischemic attack. JAMA Neurol. 2016;73(5):572-578. doi:10.1001/jamaneurol.2015.4906

38. Weimar C, Mieck T, Buchthal J, et al. Neurologic worsening during the acute phase of ischemic stroke. Arch Neurol. 2005;62 (3):393-397. doi:10.1001/archneur.62.3.393

39. Lovett JK, Coull AJ, Rothwell PM. Early risk of recurrence by subtype of ischemic stroke in population-based incidence studies. Neurology. 2004;62(4):569-573. doi:10.1212/01.WNL.0000110311.09970.83

40. Schonewille WJ, Wijman CA, Michel P, et al. Treatment and outcomes of acute basilar artery occlusion in the Basilar Artery International Cooperation Study (BASICS): a prospective registry study. Lancet Neurol. 2009;8(8):724-730. doi:10.1016/S14744422(09)70173-5

41. Toyoda K, Hirano T, Kumai Y, Fujii K, Kiritoshi S, Ibayashi S. Bilateral deafness as a prodromal symptom of basilar artery occlusion. J Neurol Sci. 2002;193(2):147-150. doi:10.1016/ S0022-510X(01)00663-3

42. Soteriades ES, Evans JC, Larson MG, et al. Incidence and prognosis of syncope. $N$ Engl J Med. 2002;347(12):878-885. doi:10.1056/NEJMoa012407

43. Sadiq W, Subhan M. Ischemic stroke of midbrain and cerebellum involving reticular activating system. Cureus. 2017;9(9):e1637.

44. Maarsingh OR, Dros J, Schellevis FG, et al. Causes of persistent dizziness in elderly patients in primary care. Ann Fam Med. 2010;8(3):196-205. doi:10.1370/afm.1116
45. Kroenke K, Lucas CA, Rosenberg ML, et al. Causes of persistent dizziness. A prospective study of 100 patients in ambulatory care. Ann Intern Med. 1992;117(11):898-904. doi:10.7326/0003-4819117-11-898

46. Saber Tehrani AS, Kattah JC, Kerber KA, et al. Diagnosing stroke in acute dizziness and vertigo: pitfalls and pearls. Stroke. 2018;49 (3):788-795. doi:10.1161/STROKEAHA.117.016979

47. Newman-Toker DE, Cannon LM, Stofferahn ME, Rothman RE, Hsieh YH, Zee DS. Imprecision in patient reports of dizziness symptom quality: a cross-sectional study conducted in an acute care setting. Mayo Clin Proc. 2007;82(11):1329-1340. doi: $10.4065 / 82.11 .1329$

48. Newman-Toker DE, Edlow JA. TiTrATE: a novel, evidence-based approach to diagnosing acute dizziness and vertigo. Neurol Clin. 2015;33(3):577-599. doi:10.1016/j.ncl.2015.04.011

49. Kattah JC, Talkad AV, Wang DZ, Hsieh YH, Newman-Toker DE. HINTS to diagnose stroke in the acute vestibular syndrome: three-step bedside oculomotor examination more sensitive than early MRI diffusion-weighted imaging. Stroke. 2009;40 (11):3504-3510. doi:10.1161/STROKEAHA.109.551234

50. Newman-Toker DE, Perry JJ. Acute diagnostic neurology: challenges and opportunities. Acad Emerg Med. 2015;22(3):357-361. doi:10.1111/acem.12614

51. Medford-Davis LN, Singh H, Mahajan P. Diagnostic decisionmaking in the emergency department. Pediatr Clin North Am. 2018;65(6):1097-1105. doi:10.1016/j.pcl.2018.07.003

52. Amarenco P, Lavallee PC, Labreuche J, et al. One-year risk of stroke after transient ischemic attack or minor stroke. $N$ Engl $J$ Med. 2016;374(16):1533-1542. doi:10.1056/NEJMoa1412981

53. Esenwa C, Gutierrez J. Secondary stroke prevention: challenges and solutions. Vasc Health Risk Manag. 2015;11:437-450. doi:10.2147/VHRM.S63791

54. Brigo F, Ragnedda G, Canu P, Nardone R. Synkinetic wrist extension in distinguishing cortical hand from radial nerve palsy. Pract Neurol. 2018;18(6):520-521. doi:10.1136/practneurol-2018-001971

55. Ricarte IF, Figueiredo MM, Fukuda TG, Pedroso JL, Silva GS. Acute foot drop syndrome mimicking peroneal nerve injury: an atypical presentation of ischemic stroke. $J$ Stroke Cerebrovasc Dis. 2014;23(5):1229-1231. doi:10.1016/j.jstrokecerebro vasdis.2013.07.009

56. Kaykisiz EK, Unluer EE. An unexpected reason for isolated foot drop: acute stroke. Pak J Med Sci. 2017;33(5):1288-1290. doi:10.12669/pjms.335.13593

57. Miller TM, Johnston SC. Should the Babinski sign be part of the routine neurologic examination? Neurology. 2005;65(8):11651168. doi:10.1212/01.wnl.0000180608.76190.10

58. Qu JF, Chen YK, Luo GP, et al. Does the Babinski sign predict functional outcome in acute ischemic stroke? Brain Behav. 2020;10(4):e01575. doi:10.1002/brb3.1575

59. Amarenco P, Lavallee PC, Monteiro Tavares L, et al. Five-year risk of stroke after TIA or minor ischemic stroke. $N$ Engl J Med. 2018;378(23):2182-2190. doi:10.1056/NEJMoa1802712

60. Easton JD, Saver JL, Albers GW, et al. Definition and evaluation of transient ischemic attack: a scientific statement for healthcare professionals from the American heart association/American stroke association stroke council; Council on cardiovascular surgery and anesthesia; Council on cardiovascular radiology and intervention; Council on cardiovascular nursing; and the Interdisciplinary council on peripheral vascular disease. The American academy of neurology affirms the value of this statement as an educational tool for neurologists. Stroke. 2009;40 (6):2276-2293. doi:10.1161/STROKEAHA.108.192218

61. Fisher CM. Transient ischemic attacks. $N$ Engl J Med. 2002;347 (21):1642-1643. doi:10.1056/NEJMp020129 
62. Castle J, Mlynash M, Lee K, et al. Agreement regarding diagnosis of transient ischemic attack fairly low among stroke-trained neurologists. Stroke. 2010;41(7):1367-1370. doi:10.1161/ STROKEAHA.109.577650

63. Coutts SB, Moreau F, Asdaghi N, et al. Rate and prognosis of brain ischemia in patients with lower-risk transient or persistent minor neurologic events. JAMA Neurol. 2019;76(12):1439-1445. doi:10.1001/jamaneurol.2019.3063

64. Lavallee PC, Cabrejo L, Labreuche J, et al. Spectrum of transient visual symptoms in a transient ischemic attack cohort. Stroke. 2013;44(12):3312-3317. doi:10.1161/STROKEAHA.113.002420

65. Lavallee PC, Sissani L, Labreuche J, et al. Clinical significance of isolated atypical transient symptoms in a cohort with transient ischemic attack. Stroke. 2017;48(6):1495-1500. doi:10.1161/ STROKEAHA.117.016743

66. Otlivanchik O, Liberman AL. Migraine as a stroke mimic and as a stroke chameleon. Curr Pain Headache Rep. 2019;23(9):63. doi:10.1007/s11916-019-0801-1

67. Dolmans LS, Lebedeva ER, Veluponnar D, et al. Diagnostic accuracy of the explicit diagnostic criteria for transient ischemic attack: a validation study. Stroke. 2019;50(8):2080-2085. doi:10.1161/STROKEAHA.119.025626

68. Lebedeva ER, Gurary NM, Gilev DV, Christensen AF, Olesen J. Explicit diagnostic criteria for transient ischemic attacks to differentiate it from migraine with aura. Cephalalgia. 2018;38 (8):1463-1470. doi:10.1177/0333102417736901

69. Goldstein JN, Camargo CA, Pelletier AJ, Edlow JA. Headache in United States emergency departments: demographics, work-up and frequency of pathological diagnoses. Cephalalgia. 2006;26 (6):684-690. doi:10.1111/j.1468-2982.2006.01093.x

70. Koudstaal PJ, van Gijn J, Kappelle LJ. Headache in transient or permanent cerebral ischemia. Dutch TIA Study Group. Stroke. 1991;22(6):754-759. doi:10.1161/01.STR.22.6.754

71. Newman-Toker DE, Moy E, Valente E, Coffey R, Hines AL. Missed diagnosis of stroke in the emergency department: a cross-sectional analysis of a large population-based sample. Diagnosis (Berl). 2014;1(2):155-166. doi:10.1515/dx-2013-0038

72. Dubosh NM, Edlow JA, Goto T, Camargo CA, Hasegawa K. Missed serious neurologic conditions in emergency department patients discharged with nonspecific diagnoses of headache or back pain. Ann Emerg Med. 2019;74(4):549-561. doi:10.1016/j. annemergmed.2019.01.020

73. Kurth T, Diener HC. Migraine and stroke: perspectives for stroke physicians. Stroke. 2012;43(12):3421-3426. doi:10.1161/ STROKEAHA.112.656603

74. Arnold M. Headache classification committee of the international headache society (IHS) the international classification of headache disorders, 3rd edition. Cephalalgia. 2018;38(1):1-211.

75. Milhaud D, Bogousslavsky J, van Melle G, Liot P. Ischemic stroke and active migraine. Neurology. 2001;57(10):1805-1811. doi:10.1212/WNL.57.10.1805

76. Sondergaard CB, Nielsen JE, Hansen CK, Christensen H. Hereditary cerebral small vessel disease and stroke. Clin Neurol Neurosurg. 2017;155:45-57. doi:10.1016/j.clineuro.2017.02.015

77. Dutra LA, de Souza AW, Grinberg-Dias G, Barsottini OG, Appenzeller S. Central nervous system vasculitis in adults: an update. Autoimmun Rev. 2017;16(2):123-131. doi:10.1016/j. autrev.2016.12.001

78. Bigal ME, Kurth T, Santanello N, et al. Migraine and cardiovascular disease: a population-based study. Neurology. 2010;74 (8):628-635. doi:10.1212/WNL.0b013e3181d0cc8b

79. Liberman AL, Lu J, Wang C, Cheng NT, Moncrieffe K, Lipton RB. Factors associated with hospitalization for ischemic stroke and TIA following an emergency department headache visit. Am J Emerg Med. 2020. doi:10.1016/j.ajem.2020.10.082
80. Liberman AL, Wang C, Friedman BW, et al. Head computed tomography during emergency department treat-and-release visit for headache is associated with increased risk of subsequent cerebrovascular disease hospitalization. Diagnosis. 2020. doi:10.1515/dx-2020-0082

81. Park J. Movement disorders following cerebrovascular lesion in the basal ganglia circuit. J Mov Disord. 2016;9(2):71-79. doi:10.14802/jmd.16005

82. Rincon F, Sacco RL. Secondary stroke prevention. $J$ Cardiovasc Nurs. 2008;23(1):34-41. doi:10.1097/01. JCN.0000305059.81000.d3

83. Kumral E, Bayam FE, Erdogan CE. Limb shaking transient ischemic attacks: a follow-up of 28 patients. Rev Neurol. 2020;176(7-8):587-591. doi:10.1016/j.neurol.2019.12.001

84. Baumgartner RW, Baumgartner I. Vasomotor reactivity is exhausted in transient ischaemic attacks with limb shaking. $J$ Neurol Neurosurg Psychiatry. 1998;65(4):561-564. doi:10.1136/ jnnp.65.4.561

85. Tatemichi TK, Young WL, Prohovnik I, Gitelman DR, Correll JW, Mohr JP. Perfusion insufficiency in limb-shaking transient ischemic attacks. Stroke. 1990;21(2):341-347. doi:10.1161/01. STR.21.2.341

86. Furlan AJ, Whisnant JP, Kearns TP. Unilateral visual loss in bright light. An unusual symptom of carotid artery occlusive disease. Arch Neurol. 1979;36(11):675-676. doi:10.1001/ archneur.1979.00500470045007

87. Graff-Radford J, Rubin MN, Jones DT, et al. The alien limb phenomenon. J Neurol. 2013;260(7):1880-1888. doi:10.1007/ s00415-013-6898-y

88. Olszewska DA, McCarthy A, Murray B, Magennis B, Connolly S, Lynch T. A wolf in sheep's clothing: an "Alien Leg" in corticobasal syndrome. Tremor Other Hyperkinet Mov. 2017;7:455. doi:10.5334/tohm.358

89. Feinberg TE, Schindler RJ, Flanagan NG, Haber LD. Two alien hand syndromes. Neurology. 1992;42(1):19-24. doi:10.1212/ WNL.42.1.19

90. Wenning GK, Litvan I, Jankovic J, et al. Natural history and survival of 14 patients with corticobasal degeneration confirmed at postmortem examination. J Neurol Neurosurg Psychiatry. 1998;64(2):184-189. doi:10.1136/jnnp.64.2.184

91. Zukić S, Sinanović O, Zonić L, Hodžić R, Mujagić S, Smajlović E. Anton's syndrome due to bilateral ischemic occipital lobe strokes. Case Rep Neurol Med. 2014;2014:1-4. doi:10.1155/ 2014/474952

92. McGlynn SM, Schacter DL. Unawareness of deficits in neuropsychological syndromes. J Clin Exp Neuropsychol. 1989;11 (2):143-205. doi:10.1080/01688638908400882

93. Greene JDW. Apraxia, agnosias, and higher visual function abnormalities. Neurol Pract. 2005;76(suppl 5):v25-v34.

94. Buyukgol H, Ilik F, Ertem DH. Evaluation of the clinical features, management, and prognoses of patients with Charles Bonnet syndrome. J Nerv Ment Dis. 2019;207(12):1045-1047. doi:10.1097/NMD.0000000000001101

95. Pang L. Hallucinations experienced by visually impaired: Charles Bonnet syndrome. Optom Vis Sci. 2016;93(12):1466-1478. doi:10.1097/OPX.0000000000000959

96. Chen JJ. Diagnosis and treatment of psychiatric comorbidity in a patient with Charles Bonnet syndrome. Case Rep Psychiatry. 2014;2014:1-8. doi:10.1155/2014/195847

97. Tekgol Uzuner G, Ubur A, Erten M, Uzuner N. A rare clinical antity; Pure Gerstmann syndrome. J Stroke Cerebrovasc Dis. 2020;29(10):105161. doi:10.1016/j.jstrokecerebrovasdis.20 20.105161

98. Ardila A. Gerstmann syndrome. Curr Neurol Neurosci Rep. 2020;20(11):48. doi:10.1007/s11910-020-01069-9 
99. Bongiorno DM, Daumit GL, Gottesman RF, Faigle R. Comorbid psychiatric disease is associated with lower rates of thrombolysis in ischemic stroke. Stroke. 2018;49(3):738-740. doi:10.1161/ STROKEAHA.117.020295

100. Zuflacht JP, Shao Y, Kronish IM, et al. Psychiatric hospitalization increases short-term risk of stroke. Stroke. 2017;48(7):17951801. doi:10.1161/STROKEAHA.116.016371

101. Liberman AL, Prabhakaran S, Newman-Toker DE. Letter by Liberman et al Regarding Article, "Psychiatric hospitalization increases short-term risk of stroke”. Stroke. 2017;48(9):e260. doi:10.1161/STROKEAHA.117.018391

102. Croskerry P. The importance of cognitive errors in diagnosis and strategies to minimize them. Acad Med. 2003;78(8):775-780. doi:10.1097/00001888-200308000-00003

103. Sherbino J, Kulasegaram K, Howey E, Norman G. Ineffectiveness of cognitive forcing strategies to reduce biases in diagnostic reasoning: a controlled trial. CJEM. 2014;16(1):34-40. doi: $10.2310 / 8000.2013 .130860$
104. Zwaan L, Monteiro S, Sherbino J, Ilgen J, Howey B, Norman G. Is bias in the eye of the beholder? A vignette study to assess recognition of cognitive biases in clinical case workups. BMJ Qual Saf. 2017;26(2):104-110. doi:10.1136/bmjqs-2015-005014

105. Ely JW, Graber ML, Croskerry P. Checklists to reduce diagnostic errors. Acad Med. 2011;86(3):307-313. doi:10.1097/ ACM.0b013e31820824cd

106. Ely JW, Graber ML. Preventing diagnostic errors in primary care. Am Fam Physician. 2016;94(6):426-432.

107. Trowbridge RL. Twelve tips for teaching avoidance of diagnostic errors. Med Teach. 2008;30(5):496-500. doi:10.1080/ 01421590801965137

108. Ely JW, Graber MA. Checklists to prevent diagnostic errors: a pilot randomized controlled trial. Diagnosis (Berl). 2015;2 (3):163-169. doi:10.1515/dx-2015-0008

109. Huang GC, Kriegel G, Wheaton $\mathrm{C}$, et al. Implementation of diagnostic pauses in the ambulatory setting. BMJ Qual Saf. 2018;27(6):492-497. doi:10.1136/bmjqs-2017-007192

\section{Publish your work in this journal}

Neuropsychiatric Disease and Treatment is an international, peerreviewed journal of clinical therapeutics and pharmacology focusing on concise rapid reporting of clinical or pre-clinical studies on a range of neuropsychiatric and neurological disorders. This journal is indexed on PubMed Central, the 'PsycINFO' database and CAS, and is the official journal of The International Neuropsychiatric Association (INA). The manuscript management system is completely online and includes a very quick and fair peer-review system, which is all easy to use. Visit http://www.dovepress.com/testimonials.php to read real quotes from published authors. 